\title{
Comparison of a 1-day and a 2-day protocol for lymphatic mapping and sentinel lymph node biopsy in patients with nonpalpable breast cancer
}

\author{
S. van Esser • M. Hobbelink • J. W. Van Isselt • \\ W. P. Th. M. Mali • I. H. M. Borel Rinkes • \\ R. van Hillegersberg
}

Received: 24 November 2008 / Accepted: 2 March 2009/Published online: 25 March 2009

(C) The Author(s) 2009. This article is published with open access at Springerlink.com

\begin{abstract}
Purpose To compare the identification rate of the sentinel node in a 1-day protocol versus a 2-day protocol in patients with a nonpalpable breast carcinoma.

Methods In the 1-day protocol an average dose of $120 \mathrm{MBq}$

${ }^{99 \mathrm{~m}} \mathrm{Tc}$-nanocolloid was injected intratumorally on the day of surgery, and in the 2-day protocol an average dose of $370 \mathrm{MBq}{ }^{99 \mathrm{~m}} \mathrm{Tc}-$ nanocolloid was injected intratumorally the day before surgery. Both a gamma ray detection probe and patent blue were used to locate the sentinel node.

Results In 57 of 67 patients (85\%) treated in the 1-day protocol and in 51 of 56 patients (91\%) treated in the 2-day protocol the sentinel node was detected $(p=0.311)$. Of the patients in the 1day protocol and the 2-day protocol, respectively, 18 (27\%) and $13(23 \%)$ showed metastasis $(p=0.975)$

Conclusion There was no significant difference in the identification rate of the sentinel node between the 1-day protocol and the 2-day protocol in patients diagnosed with a nonpalpable breast carcinoma.
\end{abstract}

S. van Esser · I. H. M. Borel Rinkes · R. van Hillegersberg

Department of Surgery, Medical Centre Utrecht,

P.O. Box 85500, 3508 GA Utrecht, The Netherlands

M. Hobbelink · J. W. Van Isselt - W. P. T. M. Mali

Department of Radiology, Radiotherapy and Nuclear Medicine,

Medical Centre Utrecht,

P.O. Box 85500,3508 GA Utrecht, The Netherlands

S. van Esser $(\square)$

Department of Surgical Oncology,

University Medical Center Utrecht,

Heidelberglaan 100,

3584 CX Utrecht, The Netherlands

e-mail: s.vanesser@umcutrecht.nl
Keywords Sentinel node biopsy.

Nonpalpable breast cancer $\cdot$ Protocol

\section{Introduction}

Lymphatic mapping and sentinel lymph node (LN) biopsy (SLNB) are reliable staging tools in the management of patients with breast carcinoma. In particular, patients with small, often nonpalpable breast carcinoma benefit from SLNB instead of axillary dissection as only $7.8-28.5 \%$ of patients with T1a-c breast carcinoma have tumour-positive LNs [1]. Therefore, without SLNB $70-90 \%$ of axillary dissections would be redundant in this particular group. In order to avoid disabling morbidity associated with axillary dissection such as lymphoedema, a successful SLNB is of great importance.

Nonpalpable breast carcinomas are often difficult to discern at surgery. Therefore a guide wire is usually placed preoperatively in order to localize the tumour. It is suggested that a guide wire placed intra- or peritumorally could disturb the lymph drainage pattern and thereby decrease the success rate of the SLNB [2].

Currently wide differences exist in SLNB protocols used. ${ }^{99 \mathrm{~m}} \mathrm{Tc}-$ Nanocolloid radiotracer, vital dye or both are used as localizing pharmaceuticals, while injection routes include intratumoral, peritumoral, intradermal and/or subareolar injection. Injection of the localizing pharmaceutical can be done either pre- or intraoperatively, on the same day or the previous day [3, 4]. Lymphoscintigraphy can be performed using either the dynamic and/or the static technique [5]. In our hospital, both radiotracer and blue dye are injected intratumorally in patients diagnosed with a nonpalpable breast carcinoma. Therefore, in nonpalpable 
breast cancer, the injection of a radiotracer needs to be guided by ultrasound or stereotaxis.

Initially patients diagnosed with a nonpalpable breast carcinoma were treated according to a 2-day protocol. This protocol was chosen to increase the number of successful SLNB due to the possibility of performing late nuclear imaging [6]. The 1-day protocol was introduced to allow all patients to be treated in day-care and to lower the radiation burden. It was unclear if there was a difference in the visualization and successful surgical removal of the sentinel node between the 1-day and the 2-day protocol and if guide wire placement influenced the success of SLNB.

The aim of this study was to compare the 1-day and 2day protocols with regard to the identification rate and removal of the sentinel node in patients diagnosed with nonpalpable breast cancer.

\section{Materials and methods}

In this retrospective, nonrandomized, cohort study, 123 patients with a nonpalpable breast carcinoma treated between July 2002 and July 2008 were included. Women with newly diagnosed primary, nonpalpable invasive breast cancer undergoing tumour resection and SLNB were eligible for the study. Exclusion criteria were previous breast cancer in the same breast, pregnancy and palpable breast cancer.

Before the introduction of a 1-day protocol, 56 patients underwent SLNB according to a 2-day protocol. Patients in the 2-day protocol received a median dose of $370 \mathrm{MBq}$ radiotracer in $0.5 \mathrm{~cm}^{3}$ of water (range $80-550 \mathrm{MBq}$ ). The 1-day protocol was introduced to allow patients to be treated in day-care and to lower the radiation burden. From then on, 67 patients were included in the 1-day protocol. The first 23 patients in the 1-day protocol received a dose of $80 \mathrm{MBq}$ radiotracer. Due to low visualization rates the subsequent 44 patients received a median dose of $120 \mathrm{MBq}$ radiotracer $(80-220 \mathrm{MBq})$ in $0.5 \mathrm{~cm}^{3}$ of water. The nanocolloid radiotracer consisted of at least $95 \%$ human albumin colloid particles of $\leq 80 \mathrm{~nm}$.

In both groups the radiotracer was injected intratumorally guided by either ultrasound or stereotaxis depending on the visibility of the tumour. Patients were asked to massage their breast after injection of the tracer. Patients in the 1-day protocol underwent lymphoscintigraphic imaging after $10 \mathrm{~min}$, and 1 and 2 hours, and patients in the 2-day protocol underwent lymphoscintigraphic imaging directly after injection and the next morning before surgery to assess the migration of the radiotracer. After finding the sentinel node on the static images the skin was marked. Intraoperatively patients received a peritumoral injection of patent blue at the site of the maximum count found with the gammaprobe (Europrobe Strasbourg, France). First, the SLNB was performed and subsequently the primary tumour was excised, followed by histopathological analysis using regular $\mathrm{H} \& \mathrm{E}$ and immunohistochemical staining. In case of failure of the SLNB a regular axillary dissection was carried out.

The number of LNs seen on static imaging was compared to the number found during surgery. The hot and/or blue sentinel nodes were removed and when the exact location of the sentinel node was unclear intraoperatively the second echelon nodes were removed as well. The nodes both inside and outside the axilla were removed. The SLNB was considered successful if the tissue removed during surgery was hot and/or blue and proved to be LN tissue on histopathological assessment. The lymphoscintigraphic visualization rate and the surgical success rate were compared between the 1-day protocol and the 2day protocol. The success rate of the SLNB in patients with a guide wire placed intratumorally before injection of the radiotracer in the 1-day protocol or the 2-day protocol was compared with that in patients without a guide wire in place during administration of the radiotracer.

\section{Statistics}

Identification and removal of one or more sentinel nodes was considered a successful SLNB. The successful removal of the sentinel node, the proportion of patients with LNs on scintigraphy and the difference in success rate of SLNB in patients with a guide wire in place during injection of the radiotracer between the two protocols were compared using the chi-squared test; $p<0.05$ was considered significant.

The numbers of sentinel nodes seen on the preoperative images and number of LN removed during surgery were compared using the independent sample $t$-test; $p<0.05$ was considered significant.

\section{Results}

The baseline characteristics in both groups were comparable, except for tumour morphology (Table 1).

The visualization rate in the 1-day protocol was 61/67 $(91 \%)$ and the visualization rate in the 2-day protocol was $55 / 56(98 \% ; p=0.087)$. The mean number of nodes seen preoperatively was 1.48 (range $0-5$ ) in the 1 -day protocol and 1.88 in the 2 -day protocol $(p=0.007)$. The visualization rates in the two protocols for nodes outside the axilla were similar (Table 2).

The surgical success rate of the SLNB was 57/67 $(85 \%)$ in the 1 -day protocol and $51 / 56(91 \%)$ in the 2 day protocol $(p=0.311)$. The mean numbers of nodes removed were 1.64 (range $0-6$ ) in the 1-day protocol and 
Table 1 Baseline characteristics

\begin{tabular}{lccc}
\hline Baseline characteristics & 1-day protocol & 2-day protocol & $p$ value \\
\hline Number of patients & 67 & 56 & \\
Number of tumours & 68 & 59 & 0.955 \\
Median age (years) & 59 & 59.5 & 0.188 \\
Median size of primary (mm) & 13 & 12 & 0.006 \\
Histology & & $56(95 \%)$ & $1(1.7 \%)$ \\
Ductal & $53(77 \%)$ & $1(1.7 \%)$ & $1(1.7 \%)$ \\
Lobular & $5(7.1 \%)$ & 0 & 0.119 \\
Ductolobular & $8(11.4 \%)$ & & \\
Mucinous & $1(1.4 \%)$ & $10(18 \%)$ & \\
Papillary & $1(1.4 \%)$ & $26(44 \%)$ & \\
Quadrant tumours & & $10(18 \%)$ & \\
Retroareolar & $5(7.4 \%)$ & $6(11 \%)$ & $5(9 \%)$ \\
Lateral upper quadrant & $35(52.2 \%)$ & & \\
Medial upper quadrant & $7(10.2 \%)$ & $(14.7 \%)$ & \\
Lateral lower quadrant & $12(18.0 \%)$ & & \\
Medial lower quadrant & &
\end{tabular}

1.61 (range $0-4)$ in the 2-day protocol $(p=0.209)$. After finding LNs on preoperative static imaging, the mean numbers of LNs not found during surgery was 0.28 (range $0-3$ ) in the 1-day protocol and 0.41 (range $0-4$ ) in the 2day protocol $(p=0.664)$. The numbers of nodes found assisted by patent blue only was 4 in the 1-day protocol and 5 in the 2 -day protocol (not significant, $p=0.73$ ). The numbers of nodes found assisted by the radiotracer and/or patent blue in the 1-day protocol and in the 2-day protocol did not differ significantly (see Table 2). Of the nonvisualized sentinel nodes, $1 / 6(17 \%)$ in the 1-day protocol and $1 / 1(100 \%)$ in the 2-day protocol were found using patent blue.

Of the patients in the 1-day protocol, 49 (73\%) showed no metastasis in the sentinel node, 3 had isolated tumour cells $(4.5 \%), 3(4.5 \%)$ had micrometastases (i.e. $<2 \mathrm{~mm})$ and $12(18 \%)$ showed macrometastases (i.e. $>2 \mathrm{~mm}$ ). Of the patients in the 2-day protocol, 42 (76.4\%) showed no metastases, $1(1.8 \%)$ had isolated tumour cells, $3(6 \%)$ had micrometastases and $9(16.4 \%)$ showed macrometastases. The number of metastases in the patients in the two protocols did not differ significantly $(p=0.975)$.

In the 1-day protocol the first 23 patients had a median dose of $80 \mathrm{MBq}$ of radiotracer. After excluding these patients, the success rate of the SLNB was $88 \%$. In 29 of 67 patients $(43 \%)$ in the 1-day protocol and in 15 of the 56 patients $(26 \%)$ in the 2-day protocol, a guide wire was in place when the radiotracer was injected. As shown in Table 3, the presence of a guide wire during injection of the radiotracer did not influence the success rate of the SLNB.
Table 2 Characteristics of the sentinel lymph node procedure

\begin{tabular}{lccc}
\hline Sentinel node & 1-day protocol & 2-day protocol (\%) & $\mathrm{p}$ \\
\hline Nodes visualized by static lymphoscintigraphy & & & 0.530 \\
Axillary & $59(88 \%)$ & $53(95 \%)$ & \\
Intramammary & $2(3 \%)$ & $2(5 \%)$ & \\
Internal mammary chain & $9(13 \%)$ & $4(7 \%)$ & 0.007 \\
Mean number & 1.48 & 1.88 & 0.087 \\
Visualization rate & $61(91 \%)$ & $55(98 \%)$ & 0.524 \\
Nodes retrieved at surgery & & & \\
Hot not blue & 23 & 5 & \\
Blue not hot & 4 & 29 & 0.209 \\
Blue and hot & 31 & 1.61 & \\
Mean number & 1.64 & & \\
\hline
\end{tabular}


Table 3 Influence of the presence of a guide wire on the success rate of SLNB

\begin{tabular}{lllll}
\hline Protocol & Guide wire & No guide wire & Total & $p$ value \\
\hline 1-day protocol & $23 / 29(80 \%)$ & $34 / 38(89 \%)$ & $57 / 67(85 \%)$ & 0.247 \\
2-day protocol & $14 / 15(93 \%)$ & $37 / 41(90 \%)$ & $51 / 56(91 \%)$ & 0.720 \\
Total & $37 / 44(84)$ & $71 / 79(90 \%)$ & $108 / 123(88 \%)$ & \\
$p$ value & 0.228 & 0.910 & & \\
\hline
\end{tabular}

\section{Discussion}

In this retrospective comparison of two historical cohorts of patients who underwent lymphoscintigraphy in a 1-day or a 2-day protocol we did not observe statistically significant differences in the identification and removal of the sentinel nodes. No significant difference in location of the sentinel node was found between the two protocols. Moreover, the presence of a guide wire when injecting the radiotracer did not influence the success rate of the SLNB.

There were some weaknesses in this study. A relatively small number of patients were retrospectively included. Therefore there are some possible confounders that could have influenced the results. In both protocols patients had a guide wire in place during injection of the radiotracer. It has been suggested that the presence of a guide wire can disturb the regular draining pattern and radiotracer can leak past the guide wire [2]. The numbers of guide wires in place during injection of radiotracer were comparable between the two groups. We could not demonstrate a negative influence of a guide wire placed intratumorally before injection of the radiotracer.

Next, the dosage of radiotracer injected in the 1-day protocol differed. It is suggested that a mean dose of at least $90 \mathrm{MBq}$ radiotracer is needed for adequate lymphoscintigraphy $[7,8]$. Possibly the higher dose of radiotracer, after excluding the first 23 patients in the 1-day protocol, resulted in the observed higher success rate of SLNB as is suggested in the literature [9]. Nevertheless, this study was nonrandomized and firm conclusions on this subject are not justified. In order to facilitate an adequate injection volume in both groups the total injected volume was $0.5 \mathrm{~cm}^{3}$. Therefore, the dilution of the radiotracer was different in the two protocols. Although there is no consensus on the volume and dilution in the current literature, the difference in dilution could have influenced the drainage patterns and speed [10].

The patients treated in the 1-day protocol underwent lymphoscintigraphy up to $2 \mathrm{~h}$ after injection. In the literature, imaging up to $4 \mathrm{~h}$ after injection is described. Possibly, late imaging could increase the number of visualized nodes, and thereby improve the success rate of the SLNB. Finally, the success rates found in this study were lower than the rates of $90-99 \%$ reported in the literature [8, 11-13].
To our knowledge, this is the first study to compare success rates between a 1-day and a 2-day protocol in nonpalpable breast cancer. Furthermore, this study provides a concise answer to a practical question. Studies performed so far included both patients with palpable and nonpalpable breast cancer. Although the protocols in these studies differed (i.e. particle size and injection region) they also showed no differences between a 1-day and a 2-day protocol [6-9]. As has been shown previously, the combination of a radiotracer preoperatively and dye injection peroperatively was superior to the use of radiotracer or dye only [3, 14]. In our study, in the 1-day protocol in four patients $(6 \%)$ and in the 2-day protocol in five patients $(9 \%)$, the sentinel node was found with patent blue only. This indicates the importance of the combination technique. In cases of non-visualization preoperatively, surgeons can still rely on the patent blue [15].

The site of injection of the radiotracer is a matter of discussion. In our protocol the radiotracer was injected intratumorally, using either ultrasound or stereotactic guidance. As reported by others, intra-/peritumoral injection of the radiotracer and the patent blue is as reliable as subareolar injection [16]. After intratumoral injection, more of the internal mammary chain LNs can be found thereby affecting the adjuvant therapeutic plan and possibly long-term survival $[17,18]$. In addition, injecting intratumorally allows lumpectomy with the radioguided occult lesion localization (ROLL) technique in the same procedure [19-21].

The use of a 1-day protocol or a 2-day protocol is often a choice based on local preferences driven by logistic considerations. In a 2-day protocol multiple patients can be scheduled for lymphoscintigraphic imaging and sentinel node procedures in the operating room. The advantage of the 1-day protocol is that patients only require one hospital visit and the radiation dose is minimized. Lowering the dose of radiotracer from $370 \mathrm{MBq}$ in the 2-day protocol to $120 \mathrm{MBq}$ in the 1-day protocol represents a threefold reduction in the radiation burden for both patients and physicians.

In conclusion, there was no significant difference in the success rate of SLNB in patients diagnosed with a nonpalpable breast carcinoma between those treated in a 1-day protocol and those treated in a 2-day protocol. In our hospital currently all patients diagnosed with a nonpalpable 
breast carcinoma are treated on a day-care basis using the 1day protocol.

Open Access This article is distributed under the terms of the Creative Commons Attribution Noncommercial License which permits any noncommercial use, distribution, and reproduction in any medium, provided the original author(s) and source are credited.

\section{References}

1. Barone JE, Tucker JB, Perez JM, Odom SR, Ghevariya V. Evidence-based medicine applied to sentinel lymph node biopsy in patients with breast cancer. Am Surg 2005;71:66-70.

2. Jansen JE, Bekker J, de Haas MJ, van der Weel FA, Verberne GH, Budel LM, et al. The influence of wire localisation for nonpalpable breast lesions on visualisation of the sentinel node. Eur J Nucl Med Mol Imaging 2006;33:1296-300. doi: 10.1007/s00259006-0119-1.

3. Anan K, Mitsuyama S, Kuga H, Saimura M, Tanabe Y, Suehara $\mathrm{N}$, et al. Double mapping with subareolar blue dye and peritumoral green dye injections decreases the false-negative rate of dye-only sentinel node biopsy for early breast cancer: 2-site injection is more accurate than 1 -site injection. Surgery 2006;139:624-9. doi: 10.1016/j.surg.2005.11.007.

4. Koizumi M, Koyama M, Yamashita T, Tada KI, Nishimura SI, Takahashi K, et al. Experience with intradermal injection and intradermal-plus-deep injection in the radioguided sentinel node biopsy of early breast cancer patients. Eur J Surg Oncol 2006;26:6.

5. Kaleya RN, Heckman JT, Most M, Zager JS. Lymphatic mapping and sentinel node biopsy: a surgical perspective. Semin Nucl Med 2005;35:129-34. doi: 10.1053/j.semnuclmed.2004.11.004.

6. Gutman F, Sanson A, Piquenot JM, Hitzel A, Ladonne JM, Dessogne P, et al. Intra-individual comparison of sentinel lymph node scintigraphy on the day of injection and on the following day in breast cancer. Nucl Med Commun 2006;27:5-9. doi: 10.1097/ 01.mnm.0000189781.62282.0d/.

7. Chagpar AB, Martin RC, Scoggins CR, Carlson DJ, Laidley AL, El Eid SE, et al. Factors predicting failure to identify a sentinel lymph node in breast cancer. Surgery 2005;138:56-63. doi: 10.1016/j.surg.2005.03.003.

8. Valdes-Olmos RA, Jansen L, Hoefnagel CA, Nieweg OE, Muller $\mathrm{SH}$, Rutgers EJ, et al. Evaluation of mammary lymphoscintigraphy by a single intratumoral injection for sentinel node identification. J Nucl Med 2000;41:1500-6.

9. Ansari SM, Heiba SI, Mills C, Abdel-Dayem HM. Localization of the breast sentinel node after axillary node dissection with diversion of lymphatic drainage to internal mammary lymph nodes and the importance of delayed imaging. Clin Nucl Med 2001;26:647-8. doi: 10.1097/00003072-200107000-00023.

10. Tanis PJ, Nieweg OE, Valdes Olmos RA, Kroon BB. Anatomy and physiology of lymphatic drainage of the breast from the perspective of sentinel node biopsy. J Am Coll Surg 2001;192:399-409. doi: 10.1016/S1072-7515(00)00776-6.

11. Heuts EM, van der Ent FW, van der Pol HA, Debets JM, Kengen RA, Verkeyn JM. Evaluation of early versus delayed lymphoscintigraphic imaging in detecting internal mammary sentinel lymph nodes in breast cancer: a multicenter study to establish an optimal lymphatic mapping protocol. Nucl Med Commun 2006;27:677-81. doi: 10.1097/00006231-200608000-00010.

12. Tanis PJ, Deurloo EE, Valdes Olmos RA, Rutgers EJ, Nieweg OE, Besnard AP, et al. Single intralesional tracer dose for radio-guided excision of clinically occult breast cancer and sentinel node. Ann Surg Oncol 2001;8:850-5. doi: 10.1007/s10434-001-0850-6.

13. Galimberti V, Zurrida S, Intra M, Monti S, Arnone P, Pruneri G, et al. Sentinel node biopsy interpretation: The Milan experience. Breast J 2000;6:306-9. doi: 10.1046/j.1524-4741.2000.20062.x.

14. Motomura $\mathrm{K}$, Inaji $\mathrm{H}$, Komoike $\mathrm{Y}$, Hasegawa $\mathrm{Y}$, Kasugai $\mathrm{T}$, Noguchi S, et al. Combination technique is superior to dye alone in identification of the sentinel node in breast cancer patients. J Surg Oncol 2001;76:95-9. doi: 10.1002/1096-9098(200102) 76:2<95::AID-JSO1018>3.0.CO;2-D.

15. Goyal A, Newcombe RG, Mansel RE, Chetty U, Ell P, Fallowfield $\mathrm{L}$, et al. Role of routine preoperative lymphoscintigraphy in sentinel node biopsy for breast cancer. Eur J Cancer 2005;41:23843. doi: 10.1016/j.ejca.2004.05.008.

16. Maza S, Valencia R, Geworski L, Zander A, Guski H, Winzer $\mathrm{KJ}$, et al. Peritumoural versus subareolar administration of technetium-99m nanocolloid for sentinel lymph node detection in breast cancer: preliminary results of a prospective intraindividual comparative study. Eur J Nucl Med Mol Imaging 2003;30:651-6.

17. Carcoforo P, Sortini D, Feggi L, Feo CV, Soliani G, Panareo S, et al. Clinical and therapeutic importance of sentinel node biopsy of the internal mammary chain in patients with breast cancer: a single-center study with long-term follow-up. Ann Surg Oncol 2006;13:1338-43. doi: 10.1245/s10434-006-9062-4.

18. Madsen E, Gobardhan P, Bongers V, Albregts M, De Burgmans J, Hooge $\mathrm{P}$, et al. The impact on post-surgical treatment of sentinel lymph node biopsy of internal mammary lymph nodes in patients with breast cancer. Ann Surg Oncol 2007;14:1486-92. doi: 10.1245/s10434-006-9230-6.

19. Feggi L, Basaglia E, Corcione S, Querzoli P, Soliani G, Ascanelli $\mathrm{S}$, et al. An original approach in the diagnosis of early breast cancer: use of the same radiopharmaceutical for both non-palpable lesions and sentinel node localisation. Eur J Nucl Med 2001;28:1589-96. doi: 10.1007/s002590100601.

20. van der Ploeg I, Hobbelink M, van den Bosch MA, Mali WP, Rinkes IH, van Hillegersberg R. 'Radioguided occult lesion localisation' (ROLL) for non-palpable breast lesions: a review of the relevant literature. Eur J Surg Oncol 2007;16:4.

21. Van Esser S, Hobbelink M, van der Ploeg I, Mali WP, van Diest PJ, Borel Rinkes I, et al. Radio guided occult lesion localization (ROLL) for non-palpable invasive breast cancer. J Surg Oncol 2008;98:526-9. 